\title{
Naproxen-Gelucire Nanoformulations for Improved Solubility and Dissolution Rate of Poorly Water-Soluble Drug Naproxen
}

\author{
Sandeep Patnaik ${ }^{1}$, Aditya Dileep Kurdekar², Lakshmi Adinarayana Avinash Chunduri ${ }^{1}$, \\ Chinnakoti Prathibha ${ }^{2}$, Kamisetti Venkataramaniah ${ }^{2, *}$ \\ ${ }^{1}$ Andhra Pradesh Medtech Zone, Vishakhapatnam, India \\ ${ }^{2}$ Laboratories for Nanoscience and Nanotechnology Research, Department of Physics, Sri Sathya Sai Institute of Higher Learning, \\ Prasanthinilayam, India
}

\section{Email address: \\ vrkamisetti@gmail.com (K. Venkataramaniah) \\ ${ }^{*}$ Corresponding author}

\section{To cite this article:}

Sandeep Patnaik, Aditya Dileep Kurdekar, Lakshmi Adinarayana Avinash Chunduri, Chinnakoti Prathibha, Kamisetti Venkataramaniah. Naproxen-Gelucire Nanoformulations for Improved Solubility and Dissolution Rate of Poorly Water-Soluble Drug Naproxen. Journal of Drug Design and Medicinal Chemistry. Vol. 3, No. 6, 2017, pp. 77-85. doi: 10.11648/j.jddmc.20170306.11

Received: June 23, 2017; Accepted: October 25, 2017; Published: November 23, 2017

\begin{abstract}
Naproxen-Gelucire Nanoformulations (NFs) in terms of their phase solubility behavior, physico-chemical characteristics, cytotoxicity and morphology and dissolution enhancement has been studied using the poorly water soluble drug, naproxen. The NFs were prepared via wet milling using a conventional Retsch Planetary ball mill in various ratios of drug to polymer $(1: 1,1: 2,1: 3,1: 4)$. The release rate of naproxen from various ratios of drug/polymer nanoparticles was investigated using USP paddle apparatus (type II). A comparative phase solubility of naproxen was performed in different carrier concentrations of simulated gastric fluid $(\mathrm{pH}$ 1.2) and simulated intestinal fluid ( $\mathrm{pH}$ 6.8). The highest dissolution enhancement was achieved for the formulation with ratio of $1: 4$. This is a $160 \%$ enhancement when compared to that of the pure drug. The ability of amphiphillic surfactant carriers to accelerate in vitro dissolution of poorly water-soluble drugs has been attributed to wetting, micellar solubilization, and/or deflocculation. The Korsemeyer-Peppas model most aptly fits the in vitro dissolution data and gives an insight into the possible drug release mechanisms predominated by anamolous non-Fickian diffusion. Thus, the nanoformulations studied can help improve the physicochemical characteristics of naproxen towards its dissolution enhancement and possibly will increase the oral bioavailability of the drug without any adverse cytotoxic consequences.
\end{abstract}

Keywords: Naproxen, Gelucire, Nanoformulations, Solubility, Dissolution, Bioavalability

\section{Introduction}

Naproxen is one of the most popular NSAIDs, widely administered against a wide range of inflammatory and analgesic disease conditions such as fever, inflammation, and pain related to a variety of muscular and skeletal disorders including osteoarthritis, bursitis, rheumatoid arthritis, kidney stones, ankylosing spondylitis, psoriatic arthritis, gout, menstrual cramps, tendinitis, and migraine. It was, first, introduced to the pharmaceutical market by Syntex Corporation, USA in the year 1976. It was first synthesized from the starting material 2-methoxynaphthalene (nerolin)
[1]. The cost competitiveness of the current manufacturing process of naproxen has continuously undergone several processes research and development during the past 20 years. In fact, the inflation-adjusted cost of naproxen today is only $25 \%$ of the 1974 cost. Currently, Naproxen is predicted to be one of the fastest-growing APIs among the adult systemic analgesics, is expected to reach nearly US $\$ 1$ billion in retail value sales by 2018 [2].

It is currently available in the United States (US) for both prescription use marketed by Genetech, a member of the Roche group and OTC use marketed by Bayer Healthcare LLC, Consumer Care. In addition, multiple generic versions of naproxen are currently available. Naproxen sodium is 
marketed as a nonprescription product by Bayer under the brand names Aleve ${ }^{\circledR}$ and Midol Extended Relief ${ }^{\circledR}$. Roche markets the prescription brands Naprosyn ${ }^{\circledR}$ (naproxen) and Anaprox ${ }^{\circledR}$ (naproxen sodium). Bayer also markets naproxen sodium in over 50 countries, including Australia and numerous European, South American, African, Asian and Caribbean countries. The earliest approval outside the US was in 1981. In most countries, the approved nonprescription dosing regimen for the temporary relief of aches and pains and the reduction of fever is $550 \mathrm{mg}$ or $660 \mathrm{mg}$ daily in adults and children 12 years of age and older. Although Naproxen has been on the market for a number of years, little is known about its method of inhibition.

While researchers have recently discovered how naproxen binds to the COX-2 enzyme, they have not yet been able to prove how this inhibits the enzyme's function. Observing the COX-2 naproxen complex, one can find the naproxen molecules buried deep within the protein structure. While how naproxen binds to COX-2 was recently discovered in September of 2010, no breakthroughs have yet been made as to how or why naproxen binding inhibits enzyme function. Like other NSAID medications naproxen is known to cause ulcers, bleeding, or holes in the lining of the stomach and intestines and should always be taken with a glass of water and antacid supplements.

Its usage is preferred for long duration administrations especially in patients having a greater cardiovascular risk and related complications such as heart attacks or strokes. This is because it has been known to offer low risk of such heart related complications compared to other NSAIDs. Chemically speaking, Naproxen is a propionic acid derivative. It is a member of the profen (2 -arylpropionic acid). Chemically it is also called (+) - (S) - 6 - Methoxy-alphamethyl-2-naphthaleneacetic acid or $\mathrm{S})$ - (+) - 2 - (6Methoxy-2-naphthyl) propionic acid.

The API is pale white in color and is an odorless, crystalline substance. Though a very old drug, it is highly lipophillic and practically insoluble in aqueous media. The drug, when orally administered, has quite some undesirable side effects like hemorrhage and ulceration of the stomach. And as a consequence of its scarce wettability and very poor water-solubility $\left(0.025 \mathrm{mg} / \mathrm{ml}\right.$ at $\left.25^{\circ} \mathrm{C}\right)$, it exhibits low and/or variable bioavailability after oral administration. Several approaches have been conducted in order to adequately improve the naproxen dissolution properties, low and/or variable bioavailability after oral administration. An improved naproxen formulation with the quick drug release pattern could be exceedingly useful in the treatment of inflammatory and painful states of the body, like rheumatoid arthritis.

In order to tackle this issue, solid dispersions with polyethylene glycol [3] or polyvinylpyrrolidone [4] or complexation with cyclodextrins $[5,6]$ and liquisolid technique [7] have been reported. In fact, the first bicomponent formulations of the drug involved complexation with 2-hydroxylpropyl- $\beta$-cyclodextrin. Lee et al [8] have reported these complexes to have increased dissolution characteristics as well as decreased gastrointestinal toxicity when administered orally. Several polymers that have been used to dose naproxen include HPMC and PVP have also been demonstrated to improve the dissolution characteristics of naproxen. Binary co-ground mixtures with drugs like cimetidine [9] and ibuprofen [10] have also been explored for improved solubility of naproxen.

Formulation of naproxen-PLGA nanoparticles by using the single emulsion-solvent evaporation/extraction process have been reported by Javadzadeh et al [11]. They were able to improve the physicochemical characteristics of the drug and speculated an increase the anti-inflammatory effects of drug following its ocular or intra-joint administration. Liversidge et al [12] have demonstrated using in vivo rat models that by reducing drug particle size to $270 \mathrm{~nm}$ and stabilizing the particles in suspension with pluronic F-68, the gastric irritation induced by oral administration of naproxen decreased, while the rate of absorption increased. The increase in the rate of absorption was attributed to an increase in surface area for dissolution for the Nanocrystal formulation.

Nanosuspensions have been reported to be advantageous due to the features such as easy industrial scalability, economic viability, high drug loading efficiency, and low excipient side effects $[13,14]$. A simple top down approach explored for drug nanoformulations (NFs) is the use of a planetary ball mill to fracture the drug crystals into smaller drug particles [15-17]. The frictional forces and the impact forces caused by the planetary rotations and revolutions of the milling jar containing the drug suspension are responsible for mechanical attrition of the contents of the milling jars [16]. In this work, the usage of this very simple and easily scalable wet ball milling technology has been explored to design novel NFs of poorly soluble drug naproxen with the polymer.

Gelucire 50/13 (the number relates to its melting point and hydrophilic-lipophilic balance value) is semi-solid lipid based solubilizer, manufactured by Gattefose, France. Chemically, it is a mixture of several glycerides (mainly C16/18) and mono and diesters of PEG 1500 (stearoyl polygyceride) $[18,19]$. These polymers form water-soluble complexes with many drug molecules, depending on the chemical structure of the Active Pharma Ingradients (APIs) [20]. The objective of this study was to evaluate the characteristics of the Naproxen- NFs in terms of their phase solubility behavior, physico-chemical characteristics, cytotoxicity, and morphology and dissolution enhancement using the poorly water soluble drug, naproxen. By fitting the dissolution data into various empirical release kinetic models, an attempt to investigate the release mechanism was made.

\section{Materials and Methods}

\subsection{Synthesis of Naproxen Nanoformulations (NFs)}

The NFs were prepared via wet milling using a conventional Retsch Planetary ball mill in various ratios of 
drug to polymer $(1: 1,1: 2,1: 3,1: 4)$. Ball milling, as a nanosizing tool, offers several advantages over other top down approaches; Firstly, installation costs are generally inexpensive. Secondly, it is adaptable towards both batchwise as well as continuous modes of operation. Planetary ball mills are mainly laboratory scale manufacturing equipment used for micronization or nanosization of a variety of powders such as ceramics, drugs, glass, silicates, etc. The Retsch planetary ball mill consists of a grinding jar positioned unconventionally on a sun wheel. This sun wheel moves in a direction opposite to that of the grinding jar. The grinding balls (agate balls, dia $10 \mathrm{~mm}$ ) in the milling jar are subjected to superimposed rotational movements, (Figure 1) also known as the Coriolis forces. Due to the difference in speed between the agate milling balls and the grinding jar, an interaction between frictional and impact forces results. The interplay between the resultant forces produces the high and dynamic energy which results in effective size reduction [21].

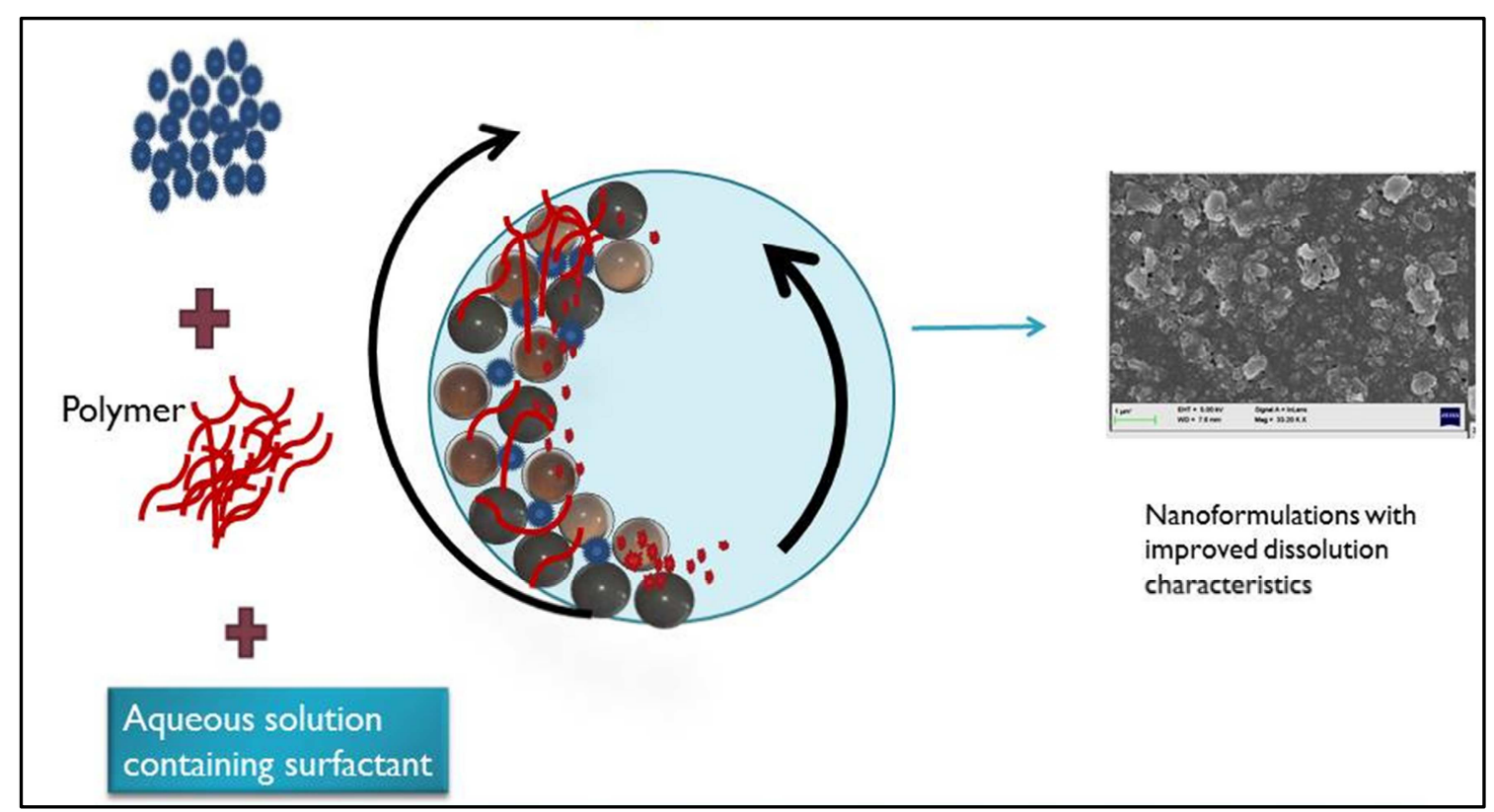

Figure 1. Schematic of Aqueous Ball Milling used to synthesize the nanoformulations.

The drug and polymer (in the required ratios) were introduced into an agate milling chamber containing $1 \mathrm{~mm}$ agate balls. $40 \mathrm{~mL}$ of $0.5 \%$ aqueous solution of Tween 80 was added to fill the chamber. Regular breaks of 5 minutes were provided after every 15 minutes of milling to avoid overheating caused due to the high energy involved in the milling process. The high shear force generated by the collision of the agate balls with the solid drug particles fractures the drug crystals into smaller particles and thus nanosuspensions were formed [17].

The nanosuspensions thus formed by co-milling were lyophilized for about 24 hours and gently powdered to obtain free flowing powders. To protect the nanoparticles from damage, due to ice formation and to minimize the particle size growth during lyophillization, mannitol ( $0.1 \%$ by weight $)$ was added as a cryoprotectant prior to lyophillization. The several formulations of naproxen designed and studied are listed in Table 1.

Table 1. Different nanoformulations of Gelucire 50/13 studied.

\begin{tabular}{llll}
\hline S. No & $\begin{array}{l}\text { Formulation } \\
\text { Label }\end{array}$ & $\begin{array}{l}\text { Drug - Carrier } \\
\text { ratio }\end{array}$ & $\begin{array}{l}\text { Percentage of } \\
\text { carrier }\end{array}$ \\
\hline 1 & NG 1 & $1: 1$ & 50 \\
2 & NG 2 & $1: 2$ & 66 \\
3 & NG 3 & $1: 3$ & 75 \\
4 & NG 4 & $1: 4$ & 80 \\
\hline
\end{tabular}

\subsection{Phase Solubility Studies}

Phase solubility analysis is a simple and elegant technique traditionally used to assess the absolute purity of a crystalline material. It was used for the quantitative determination of the purity of a substance through the application of precise solubility measurements. Constancy of solubility, like constancy of melting temperature or other physical properties, indicate that the material is pure or is free from foreign admixture except in the unique case in which percentage composition of the substance under test is in direct ratio to solubility of respective components. Of late, the phase solubility studies have found their usage to determine the suitability of carriers for solubility enhancement and the spontaneity of the drug solubilization process in the presence of the polymers in solution [22, 23].

Phase solubility profiles of naproxen in various concentrations of the polymer were established by the method established by Higuchi and Connors [24]. The procedure used was as follows:

To Erlenmeyer flasks $(250 \mathrm{~mL})$ containing $25 \mathrm{~mL}$ of the various polymer solution $(0.1 \%, 0.25 \%, 0.5 \%, 0.75 \%$ and $1 \%$, $\mathrm{w} / \mathrm{v})$, an excess amount of drug (1g) was added. The flasks were suitably sealed and shaken at $100 \mathrm{rpm}$ in orbital shakerincubator for 48 hours at $37^{\circ} \mathrm{C}$. They were left in the incubator for another 24 hours for equilibrium to be 
established. $5 \mathrm{ml}$ of the supernatant solution was withdrawn and filtered. The amount of drug in the filtrate was photometrically analyzed spectrophotometrically at $278 \mathrm{~nm}$ for determination of the naproxen content using the calibration curve illustrated in Figure 2. The studies were carried out 5 times.

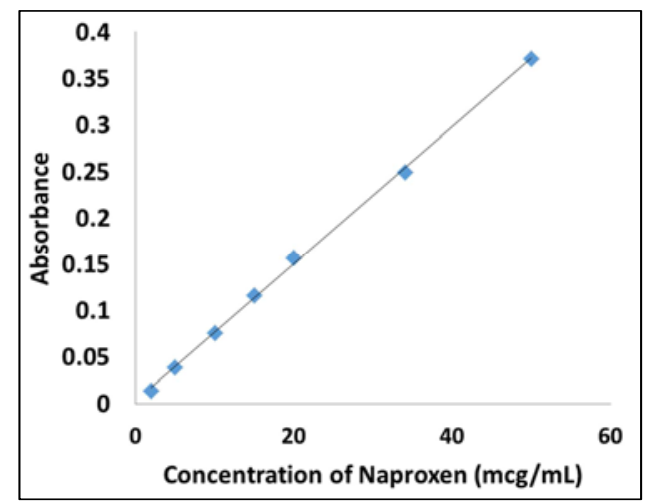

Figure 2. Calibration Curve used for spectrophotometric determination of naproxen using Beer Lambert's Law.

Gibbs free energy of transfer $\left(\Delta \mathrm{G}^{\circ}{ }_{\text {tr }}\right)$ values indicate whether the particular treatment is favorable for the solubilization of the drug in an aqueous medium [23]. The more negative the value, the more the spontaneity of the solubilization process. The $\Delta \mathrm{G}^{\circ}$ tr values of Naproxen were computed from the data obtained from phase solubility studies using the following equation $[22,23]$ :

$$
\Delta G_{t r}^{0}=-2.303 R T \log \frac{S_{0}}{S_{s}}
$$

where;

$\mathrm{S}_{\mathrm{o}}=$ molar solubility of neproxen in distilled water

$\mathrm{S}_{\mathrm{s}}=$ molar solubility of neproxen in the presence of Gelucire

$\mathrm{R}=8.31 \mathrm{JK}^{-1} \mathrm{~mol}^{-1}$

$\mathrm{T}=$ temperature in degree kelvin.

Figure 2 shows the influence of increasing concentration of the carriers on the solubility of naproxen in simulated gastric fluid (pH 1.2) and simulated intestinal fluid (pH 6.8). Pure Naproxen exhibited higher solubility in SIF (49.12 $\mu \mathrm{g} / \mathrm{mL})$ than in SGF $(2.42 \mu \mathrm{g} / \mathrm{mL})$. This could be attributed to the fact that naproxen is a weak acid with a $\mathrm{pK}_{\mathrm{a}}$ value of 4.15 . So, percentage of naproxen ionized would be much more in SIF than in SGF. The drug, thus, exhibits $\mathrm{pH}$ dependent solubility [7]. The phase solubility data show a linearly increasing trend in naproxen solubility with increasing carrier levels (Figure 2). The high solubilizing effect of the block copolymer Gelucire could be attributed to the multiple interaction sites in its chain and its surface active properties [25]. The solubility of a drug in dissolution media can be influenced by altering different physicochemical properties, like hydrophobicity/hydrophilicity, viscosity, chemical structure and polarity, etc.

Gelucires are amphiphilic pharmaceutical excipients that have been used as powerful solubilizing agents and bioavailability enhancers for orally and topically administered drugs. They are essentially saturated polyglycolized glycerides, available with variable functionalities tailor-made to establish a particular hydrophilic to lipophilic balance. For example, gelucire44/14 (lauroyl macrogolglycerides) has a hydrophilic-lipophilic balance (HLB) of 14 and a melting temperature of $44^{\circ} \mathrm{C}$, hence its name. Gelucire 44/14, thus, has interesting properties due to its unique combination of surfactants (mono and diesters of polyethylene glycol), cosurfactants (monoglycerides), and oily phase (diglycerides and triglycerides). The incorporation of gelucires in drug formulations has been reported to increase the dissolution rate of poorly soluble drugs such as aceclofenac [17], piroxicam [18] and glibenclamide [19] leading to an improved drug bioavailability

The $\Delta \mathrm{G}_{\text {tr }}^{\mathrm{o}}$ values obtained from the phase solubility curves with several carriers are listed in Table 2 . The most negative value of $\Delta \mathrm{G}_{\mathrm{tr}}^{\mathrm{o}}$ is obtained for the solution with Gelucire which is indicative of fact that the process of transfer of naproxen from the bulk medium to its aqueous solutions was most favorable.

Table 2. $\Delta G \operatorname{tr}$ (joules/mol) obtained from the phase solubility studies for the carrier Gelucire.

\begin{tabular}{lll}
\hline Percentage of Polymer $(\mathbf{W} / \mathbf{V})$ & $\mathbf{\Delta} \mathbf{G}_{\text {tr }}(\mathbf{j o u l e s} / \mathbf{m o l})$ for the different carriers at $37^{\circ} \mathbf{C}$ in SGF & $\begin{array}{l}\Delta \mathbf{G}_{\text {tr }}\left(\mathbf{j o u l e s} / \mathbf{m o l}_{\text {in }} \text { for the different carriers at } 37^{\circ} \mathbf{C}\right. \\
\text { in }\end{array}$ \\
\hline 0.1 & -2186.471 & -350.717 \\
0.25 & -4539.736 & -640.407 \\
0.5 & -5564.670 & -1093.34 \\
0.75 & -6191.089 & -1366.64 \\
1.0 & -6687.629 & -1624.05 \\
\hline
\end{tabular}

\section{Results and Discussion}

\subsection{Physico-Chemical Characteristics}

\subsubsection{Differential Scanning Calorimetry}

Figure 3 depicts the thermal behavior of the nanoformulations in comparison with the pure drug and 50/13. The DSC of naproxen showed a sharp endotherm
$\left(\mathrm{T}_{\text {onset }}=154, \mathrm{~T}_{\mathrm{M}}=157.49\right.$, and $\left.\Delta \mathrm{H}_{\text {fus }}=144.23 \mathrm{~J} / \mathrm{g}\right)$ attributed to the melting of the drug. The TA curves 50/13 depicts a broad melting endotherm at around $50^{\circ} \mathrm{C}$ which begins with a prominent decrease just as the temperature crossed the ambient conditions $\left(25^{\circ} \mathrm{C}\right)$. The TA curves of the nanosuspensions with lower polymeric ratio NG 1 shows a very weak endotherm with decreased onset drug melting point temperature. The TA curve of NG4 shows complete absence of the drug melting peak. In both the cases, the 
melting point of the polymer was observed to decrease in the presence of the drug. This could be because the drug-polymer interactions substitute the polymer-polymer interactions in the nanoformulations.

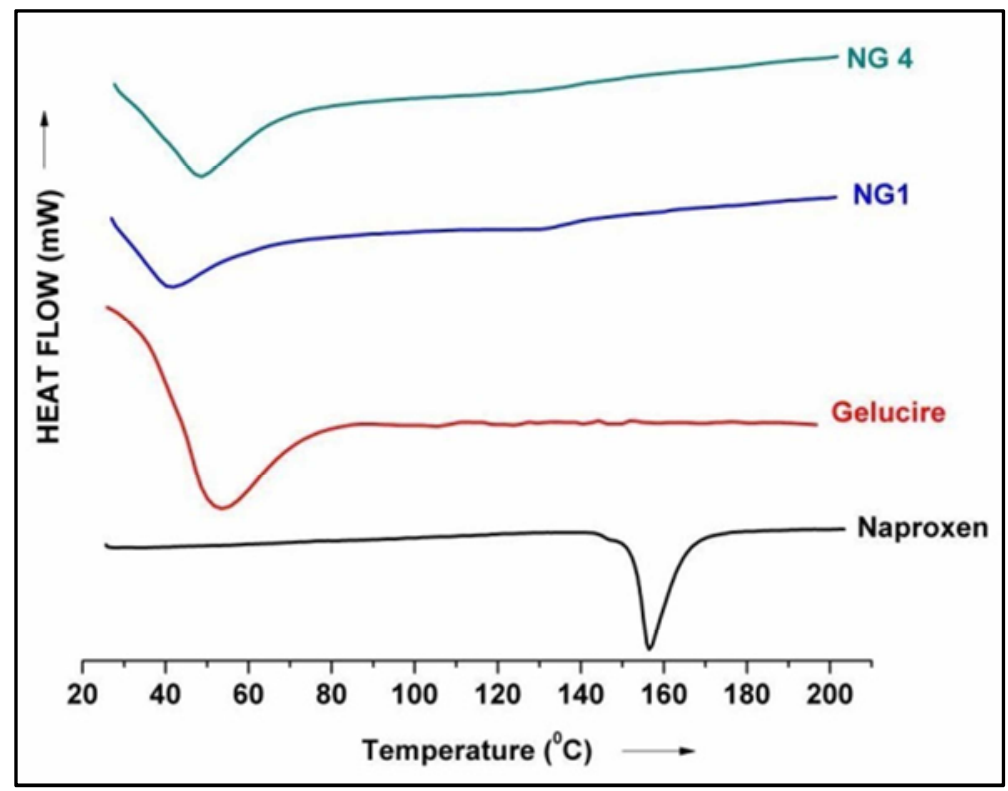

Figure 3. DSC curves of Naproxen, and the nanoformluations.

\subsubsection{FTIR Analysis}

Figure 4 shows the overlay of the FTIR spectra of naproxen and the several nanoformulations. The spectra of $50 / 13$ shows a large band between and $3100 \mathrm{~cm}^{-1}$ for free $\mathrm{OH}$ stretching vibration of its $-\mathrm{COOH}$ groups and $1739 \mathrm{~cm}^{-1}$
$(\mathrm{C}=\mathrm{O}$ stretch $)$ and several other singlet bands (1120 and 1470 $\mathrm{cm}^{-1}$ ) and a doublet peak at $965 \mathrm{~cm}^{-1}$. The reduction in the carbonyl peak in intensity in the nanoformulations could be suggestive of $\mathrm{H}$ bonding.

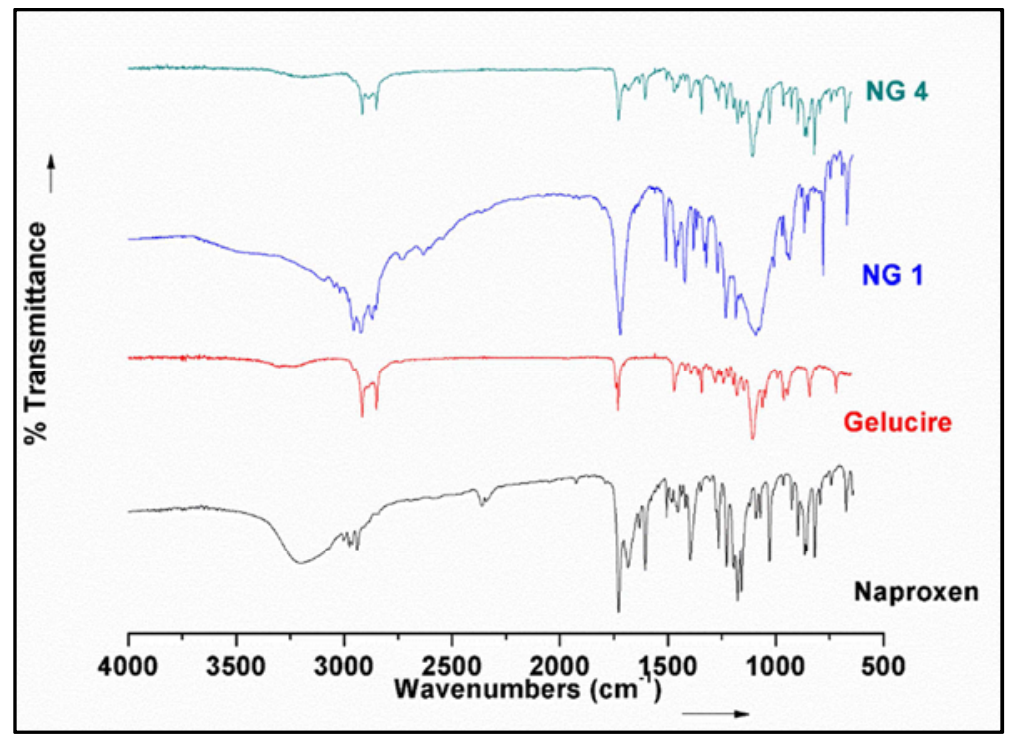

Figure 4. FTIR spectra of Naproxen and the nanoformluations.

\subsubsection{XRD Analysis}

As can be seen in Figure 5, distinct sharp peaks of naproxen were obtained at the diffraction angles 14.50, 17.73 and 27.45. The nanoformulation NG 1 showed the presence of lowered crystallinity compared to that of the pure drug. The nanoformulations NG 2 and NG 4 were completely x-ray amorphous with no crystalline peaks of the drugs at all. This is in coherence with the thermal analysis plots. It can be thus expected that naproxen is present as amorphous dispersions in the matrix of $50 / 13$. 


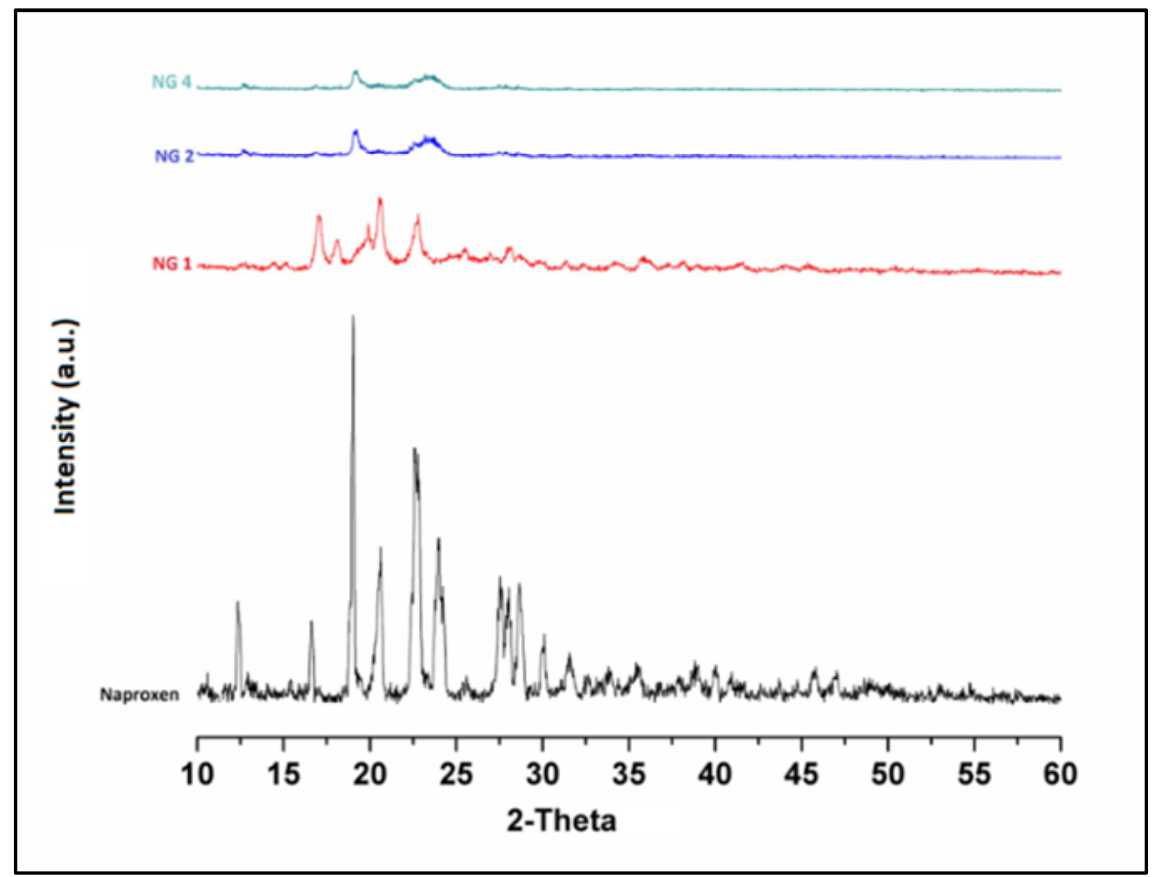

Figure 5. XRD spectra of Naproxen and its nanoformluations with Gelucire.

\subsubsection{Morphology and Particle Size}

The FESEM images (Figure. 6) of the nanformulations show that the carrier content hardly had an effect on the morphology of the nanformulations. All the formulations appear to be waxy composites with no clear distinction
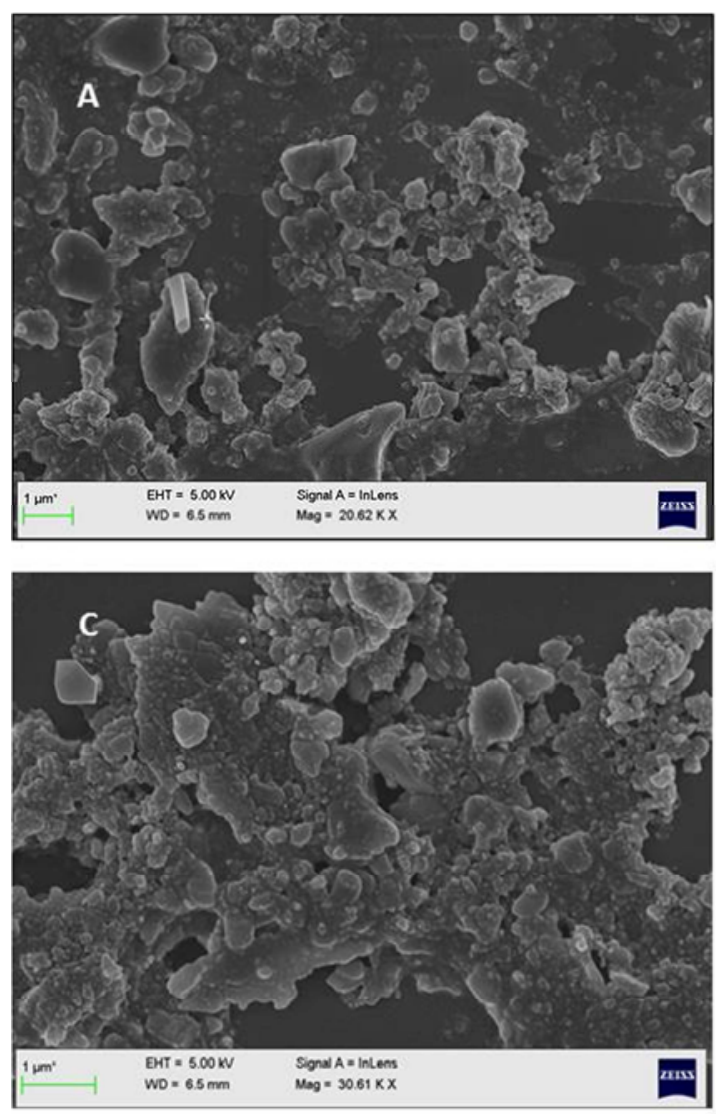

between the drug and the polymer. The carrier content hardly had an effect on the morphology of the nanformulations. The nano sized protrusions $(75-120 \mathrm{~nm})$ observed on the surface of the formulations could be responsible of the increase in the surface area and thus dissolution characteristics of naproxen.
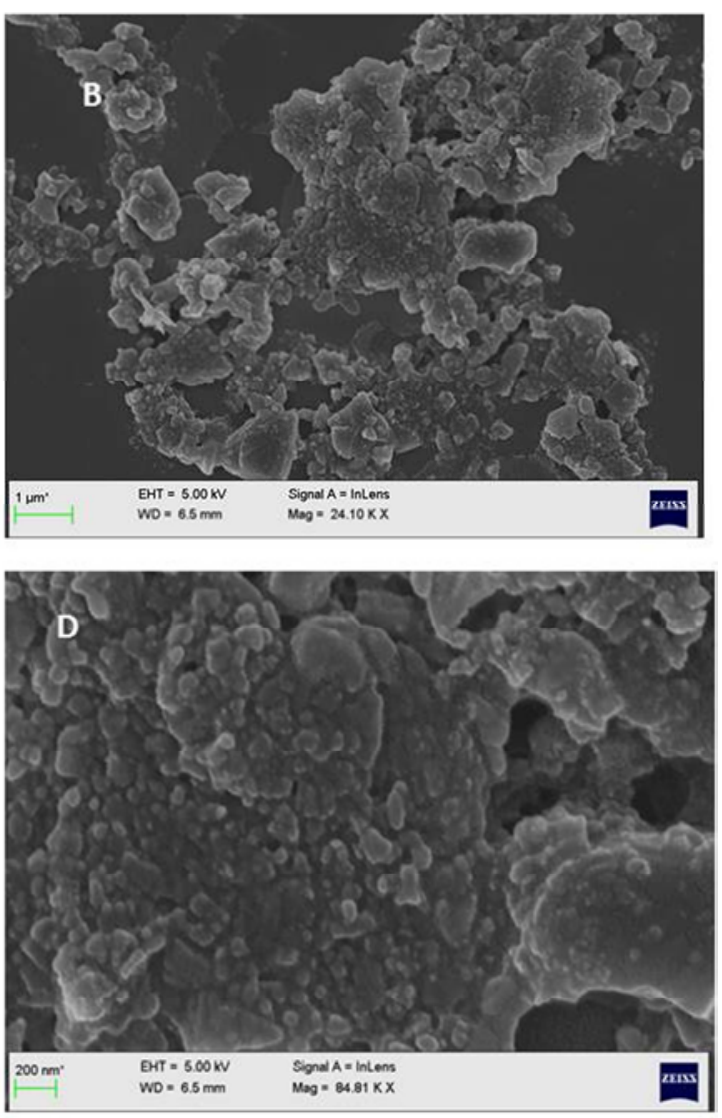

Figure 6. FESEM images of Naproxen: Nanoformulations $N G 1$ ( $A$ and $B)$ and $N G 4$ (C and D). 


\subsection{Dissolution Analysis}

The dissolution of naproxen from all the NFs of was clearly observed to be much higher than that of the pure drug in SIF (Figure 7) and was observed to increase with increasing content. During the dissolution studies, the drug particles were observed to disperse into the bulk of the medium rather quickly indicating rapid wetting property of the polymer. Since there was no decline in the supersaturation observed in case the nanoformulations of even at the end of two hours, it can be concluded that was successful in prevent the recrystallization of the drug in the given dissolution time span. The formulation NG4 was observed to get supersaturated with $95 \%$ dissolution rate of the pure drug in just 10 minutes. This was a $160 \%$ enhancement achieved when compared to the dissolution of the pure drug at 10 minutes. The high and sustainable solubility enhancement from could be attributed to micellar solubilization (amphiphillic nature of the carriers), improved wetting characteristics and reduced crystallinity of the drug in the carrier systems $[28,29]$.

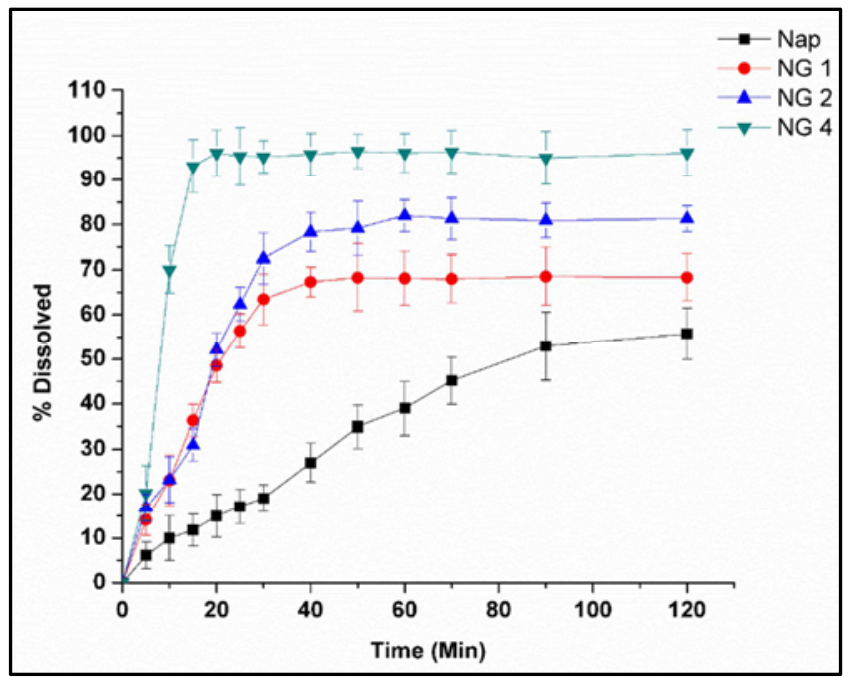

Figure 7. Comparative dissolution profiles of Naproxen and the various nanoformulations of Naproxen-Gelucire.

Table 3. Percentage Dissolution Efficiency.

\begin{tabular}{llllll}
\hline \multirow{2}{*}{ Formulation } & \multicolumn{2}{l}{ \% Dissolution Efficiency } & & & \\
\cline { 2 - 6 } & $\mathbf{5}$ min & $\mathbf{1 0}$ min & $\mathbf{2 0}$ min & 30 min & 90 min \\
\hline Pure Naproxen & 3.051 & 5.556 & 8.871 & 11.607 & 29.542 \\
NG 1 & 11.083 & 15.010 & 34.175 & 57.306 & 84.344 \\
NG 2 & 17.945 & 23.659 & 43.848 & 65.395 & 73.052 \\
NG 4 & 10.077 & 27.580 & 57.828 & 70.377 & 87.396 \\
\hline
\end{tabular}

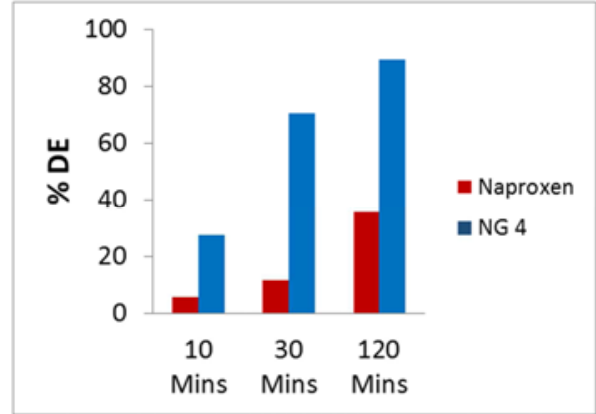

Figure 8. The comparison of Dissolution Efficiencies calculated from the dissolution plots of Naproxen and the NF NG 4.

Figure 8 and Table 3 show the \% DE values of the nanoformulation NG 4 in comparison with that of the pure drug at three different time scales representing early and late phase of dissolution. At $t=10$ early phase of dissolution, NG 4 shows an enhanced D. E value of $30 \%$. This is a 4 -fold enhancement of the efficiency compared to that of pure drug. Similarly, at $\mathrm{t}=30 \mathrm{~min}$, the $\%$ DE increased from $11 \%$ to $70 \%$. An efficiency enhancement of $157 \%$ was achieved by $\mathrm{NG}$ at $\mathrm{t}=120$ in comparison with that of the pure drug.

\subsection{Cytotoxicity Studies on Naproxen Nanoformulations}

Caco-2 cells were used as in vitro models to assess the cell viability characteristics of the carrier Gelucire and the nanoformulations of Naproxen. The assessment of cell viability was done using the tetrazolium salt based MTT assay. Duration of 12 hour exposure was selected because scintigraphic gastric transit studies in humans suggest they are physiologically relevant average and maximum exposure times, respectively, in the gastrointestinal tract. All the NFs were observed to follow the cytotoxicity trend of the polymers. Our results show that no significant decrease in cell viability was seen until $0.01 \%$ concentration of Gelucire for 12-h exposure. The NFs as well as the polymer alone had no significant effect on the viability of Caco-2 cells below $0.01 \%$ concentrations. Here it is important to note that intact intestinal membranes (in vivo) often are found to be more resistant to the cytotoxic effects of excipients than are cell culture models (in vitro). The intestine has a protective mucous layer, whereas the cell culture monolayers do not. The intestinal tissues also have more capacity to recover from trauma than the cultured cells. Hence the present NFs can be expected to show lesser cytotoxicity when subjected to in vivo studies.

The dissolution of naproxen from all the NFs with Gelucire was significantly faster than that of the pure drug in both the dissolution media. Also, increase in the polymer content in the fomulations had an enhanced effect on the dissolution of naproxen. The release from Gelucire based NFs visually revealed the tendency of the drug to leave the surface of the dissolution medium instantaneously and disperse in the bulk of the medium indicating the occurrence of rapid wetting. There was no visible decline in the 
supersaturation in case of the NFs even at the end of two hours. The high and sustainable solubility enhancement from Gelucire could be attributed to micellar solubilization and/or reduction of activity coefficient of the drug through reduction of hydrophobic interaction (s).

The Noyes-Whitney equation [20] is often used explain the dissolution results.

$$
\frac{d C}{d t}=D S \frac{\left(C_{S}-C\right)}{h}
$$

where $\mathrm{dC} / \mathrm{dt}$ is the dissolution rate, $\mathrm{D}$ is the diffusion coefficient of the dissolved drug particles, which is a parameter viscosity of the dissolution medium; $S$ represents the exposed surface area to dissolution; $h$ is the thickness of the diffusion layer, which is a parameter affected by agitation; Cs is the saturation solubility of the drug in solution in the diffusion layer, the term $\mathrm{C}$ is the concentration of the drug in the dissolution medium. Since the dissolution tests were performed under the same stirring conditions $(70 \mathrm{rpm})$ and the dissolution media was prepared with same viscosity, the parameters in the equation $\mathrm{h}$ and $\mathrm{D}$ can be assumed to be constant. Thus, the only terms affecting the dissolution rates of the nanoformulations can be assumed to be $\left(\mathrm{C}_{\mathrm{s}}-\mathrm{C}\right)$. It can, therefore, be concluded that the wettability of the drug particles were increased and the particle size decreased. The enhancement in the dissolution of the nanoformulations could thus be attributed to a combined effect of decrease in particle size of the drug as well as improved wetting characteristics of the polymer. For a comparative analysis of the drug release from the formulations, \% DE values at several times, representing the various phases of dissolution study, were computed (Table 3).

Dissolution efficiency (DE) is the area under the dissolution curve within a given range of time. Lower\%DE values observed for NG1 could probably be attributed to slow emulsification process leading to slower dissolution in the initial time periods. At the end of 120 min NG4 achieved an improvement of $160 \%$. Lower $\% \mathrm{DE}$ values observed for NG1 could probably be attributed to slow emulsification process leading to slower dissolution in the initial time periods.

\subsection{Mathematical Modeling}

The dissolution profiles from formulations have been treated with several mathematical models to describe release rates and mechanisms, their utility being dependent on the nature of dosage. When drug release rate is proportional to the drug remaining in the dosage form, dissolution can be said to follow first order release kinetics. The data obtained from the dissolution analysis were fitted into various mathematical models listed in Table 4 to describe the drug release mechanism from the different formulations.

Table 4. Mathematical Models for Studying the Drug Release Kinetics from Dissolution Profiles.

\begin{tabular}{llll}
\hline S. No & Model & Equation & Plot \\
\hline 1. & Zero order & $\ln \left(\mathrm{M}_{\mathrm{o}} / \mathrm{M}_{\mathrm{t}}\right)=\mathrm{k}_{0} \mathrm{t}$ & Cumulative amount of drug released versus time. \\
2. & First order & $\mathrm{M}_{\mathrm{o}}-\mathrm{M}_{\mathrm{t}}=\mathrm{k}_{1} \mathrm{t}$ & Log of percentage of drug remaining unreleased versus time. \\
3. & Higuchi & $\mathrm{M}_{\mathrm{t}}=\mathrm{K} \sqrt{\mathrm{t}}$ & Cumulative percent release versus square root of time. \\
4. & Hixson-Crowell & $\left(\mathrm{M}_{\mathrm{o}}\right)^{1 / 3}-\left(\mathrm{M}_{\mathrm{t}}\right)^{1 / 3}=\mathrm{k}_{1 / 3} \mathrm{t}$ & Cube root of drug percentage remaining in the matrix versus time. \\
5. & Korsemeyer-Peppas & $M_{\mathrm{t}} / M_{\infty}=\mathrm{kt}^{\mathrm{n}}$ & Log of the cumulative percent drug release versus log of time. \\
\hline
\end{tabular}

The release kinetics of a drug can be influenced by several parameters. For a poorly water-soluble drug, like naproxen, release kinetics should be predominantly guided by erosion of the matrix. The analysis of the data obtained from the dissolution studies with mathematical formulae helps relating the results as a function of the formulation characteristics. The analysis of the data has been done on some empirical drug release equations.

Table 5 lists the slopes and $\mathrm{R}^{2}$ values obtained from fitting the experimental in vitro dissolution data into the various release kinetic models. The fittings were carried out for the data obtained till 40 minutes for the quick release formulations NG4. Considering only the data points till superstauration was achieved, the data fit well into the first order and the Korsemeyer-Peppas [25] models. According to the regression values, the drug release data were observed to best fit into kinetic models in the order: Korsemeyer-Peppas $\approx$ First order $>$ Higuchi $>$ Hixon-Crowel $>$ Zero order.

Table 5. Slopes and $R^{2}$ values obtained from fitting the experimental in vitro dissolution data into the various release kinetic models.

\begin{tabular}{|c|c|c|c|c|c|c|c|c|c|c|}
\hline \multirow{2}{*}{ Formulation } & \multicolumn{2}{|c|}{ Zero Order } & \multicolumn{2}{|c|}{ First order } & \multicolumn{2}{|c|}{ Higuchi [24] } & \multicolumn{2}{|c|}{ Hixson-Crowell } & \multicolumn{2}{|c|}{ Korsemeyer-Peppas [21] } \\
\hline & Slope & $\mathbf{R}^{2}$ & Slope & $\mathbf{R}^{2}$ & Slope & $\mathbf{R}^{2}$ & Slope & $\mathbf{R}^{2}$ & Slope & $\mathbf{R}^{2}$ \\
\hline NG1 & -0.033 & 0.672 & -0.001 & 0.967 & 23.112 & 0.845 & 0.077 & 0.768 & 0.898 & 0.610 \\
\hline NG2 & -0.043 & 0.567 & -0.002 & 0.966 & 26.912 & 0.866 & 0.032 & 0.883 & 0.844 & 0.554 \\
\hline NG4 & -0.242 & 0.518 & -0.002 & 0.963 & 34.862 & 0.873 & 0.11 & 0.684 & 0.545 & 0.651 \\
\hline
\end{tabular}

This model describes the release of the drug from polymeric matrices based on the release exponent factor ' $n$ ' which is calculated as the slope when log of the percentage of the drug released is plotted versus the log of time. This $n$ value characterizes the nature of different release mechanisms for Fickian diffusion $(n=0.5)$ or non-Fickian/ anomalous release (for $0.5<\mathrm{n}<1$ ). Since the values of diffusional exponent ' $n$ ', obtained from the fitting, ranged from 0.545 to 0.966 , the release phenomena may be regarded to follow a non-fickian model. 


\section{Conclusion}

The highest dissolution enhancement was achieved for the formulation with Gelucire with ratio of 1:4. This is a $160 \%$ enhancement when compared to that of the pure drug. The ability of amphiphillic surfactant carriers to accelerate in vitro dissolution of poorly water-soluble drugs has been attributed to wetting, micellar solubilization, and/or deflocculation. Thus, the nanoformulations studied can help improve the physicochemical characteristics of Naproxen towards its dissolution enhancement and possibly will increase the oral bioavailability of the drug without any adverse cytotoxic consequences.

\section{References}

[1] Harrington P. J. \& Lodewijk, E. (1997) Twenty, Years of Naproxen Technology. Org. Process Res. Dev. 1, 72-76.

[2] Global and Chinese Naproxen (CAS22204-53-1) Industry2015 http://www.researchandmarkets.com/reports/3343235/ Global.

[3] Mura, P., Faucci, M. T., Manderioli, a, Bramanti, G. \& Parrini, P. (1999), Thermal behavior and dissolution properties of naproxen from binary and ternary solid dispersions. Drug Dev. Ind. Pharm. 25, 257-64.

[4] Mura, P., Faucci, M. T. \& Bettinetti, G. P. (2001), The influence of polyvinylpyrrolidone on naproxen complexation with hydroxypropyl-??-cyclodextrin. Eur. J. Pharm. Sci. 13, 187-194.

[5] Mura, P. et al. (2005) Interaction of naproxen with ionic cyclodextrins in aqueous solution and in the solid state. $J$. Pharm. Biomed. Anal. 37, 987-994.

[6] Mura, P. et al (2005) Solid-state characterization and dissolution properties of Naproxen-Arginine-Hydroxypropylcyclodextrin ternary system. Eur. J. Pharm. Biopharm. 59, 99-106.

[7] Tiong, N. \& Elkordy, A. A. (2009), Effects of liquisolid formulations on dissolution of naproxen. Eur. J. Pharm. Biopharm. 73, 373-384.

[8] Lee, B. J. \& Lee, J. R. (1995) Enhancement of solubility and dissolution rate of poorly water- soluble naproxen by complexation with 2-hydroxypropyl-beta- cyclodextrin. Arch. Pharm. Res. 18, 22-26.

[9] Allesø, M. et al. (2009) Enhanced dissolution rate and synchronized release of drugs in binary systems through formulation: Amorphous naproxen-cimetidine mixtures prepared by mechanical activation. J. Control. Release 136, $45-53$.

[10] Bogdanova, S., Pajeva, I., Nikolova, P., Tsakovska, I. \& Müller, B. (2005) Interactions of poly(vinylpyrrolidone) with ibuprofen and naproxen: experimental and modeling studies. Pharm. Res., 22, 806-15.
[11] Javadzadeh, Y. et al. (2010) Preparation and physicochemical characterization of naproxen-PLGA nanoparticles. Colloids Surf. B. Biointerfaces 81, 498-502.

[12] Liversidge, G. G. \& Conzentino, P. 1(995) Drug particle size reduction for decreasing gastric irritancy and enhancing absorption of naproxen in rats. Int. J. Pharm. 125, 309-313.

[13] Müller, R. H., Jacobs, C. \& Kayser, O. (2001) Nanosuspensions as particulate drug formulations in therapy: Rationale for development and what we can expect for the future. Adv. Drug Deliv. Rev. 47, 3-19.

[14] Patravale, V. B., Date, A. a \& Kulkarni, R. M.(2004) Nanosuspensions: a promising drug delivery strategy. $J$. Pharm. Pharmacol. 56, 827-40.

[15] Bhakay, A., Merwade, M., Bilgili, E. \& Dave, R. N.(2011) Novel aspects of wet milling for the production of microsuspensions and nanosuspensions of poorly watersoluble drugs. Drug Dev. Ind. Pharm. 37, 963-976.

[16] George, M. \& Ghosh, I. (2013) Identifying the correlation between drug/stabilizer properties and critical quality attributes (CQAs) of nanosuspension formulation prepared by wet media milling technology. Eur. J. Pharm. Sci. 48, 142152 .

[17] Ghosh, I. \& Michniak-Kohn, B.(2012) Design and characterization of submicron formulation for a poorly soluble drug: The effect of Vitamin e TPGS and other solubilizers on skin permeability enhancement. Int. J. Pharm. 434, 90-98.

[18] Reintjes, T.(2011) Solubility Enhancement with BASF Pharma Polymers Solubilizer Compendium. October.

[19] Patnaik, S., Aditha, S. K., Rattan, T. \& Kamisetti, V.(2015) Aceclofenac-Soluplus Nanocomposites for Increased Bioavailability. Soft Nanosci. Lett. 5, 13-20.

[20] Noyes A. A, Whitney W. R. (1897) The rate of solution of solid substances in their own solutions. J. Am. Chem. Soc. 19: 930-934.

[21] Florez-Zamora, M. I. et al. (2008) Comparative study of AlNi-Mo alloys obtained by mechanical alloying in different ball mills. Rev. Adv. Mater. Sci. 18, 301-304.

[22] Ahuja, N., Katare, O. P. \& Singh, B.(2007) Studies on dissolution enhancement and mathematical modeling of drug release of a poorly water-soluble drug using water-soluble carriers. Eur. J. Pharm. Biopharm. 65, 26-38.

[23] Maulvi, F. a. et al. (2007) Improvement of dissolution rate of aceclofenac by solid dispersion technique. Powder Technol. 207, 218-226.

[24] Higuchi, T. \& Connors, K. A. (1965) Phase-Solubility Techniques. Adv. Anal. Chem. Instrum. 4, 117-210 (1965).

[25] Korsmeyer, R. W., Gurny, R., Doelker, E., Buri, P. \& Peppas, N. A. (1983) Mechanisms of solute release from porous hydrophilic polymers. Int. J. Pharm. 15, 25-35. 